TRANSACTIONS OF THE

AMERICAN MATHEMATICAL SOCIETY

Volume 349, Number 5, May 1997, Pages 1811-1820

S 0002-9947(97)01760-1

\title{
BODIES WITH SIMILAR PROJECTIONS
}

\author{
G. D. CHAKERIAN AND E. LUTWAK
}

\begin{abstract}
Aleksandrov's projection theorem characterizes centrally symmetric convex bodies by the measures of their orthogonal projections on lower dimensional subspaces. A general result proved here concerning the mixed volumes of projections of a collection of convex bodies has the following corollary. If $K$ is a convex body in $\mathbb{R}^{n}$ whose projections on $r$-dimensional subspaces have the same $r$-dimensional volume as the projections of a centrally symmetric convex body $M$, then the Quermassintegrals satisfy $W_{j}(M) \geq W_{j}(K)$, for $0 \leq j<n-r$, with equality, for any $j$, if and only if $K$ is a translate of $M$. The case where $K$ is centrally symmetric gives Aleksandrov's projection theorem.
\end{abstract}

Let $\mathcal{C}^{n}$ denote the set of convex figures (compact, convex subsets) of Euclidean $n$-space, $\mathbb{R}^{n}$. Let $\mathcal{K}^{n}$ denote the subset of $\mathcal{C}^{n}$ consisting of the convex bodies (convex figures with non-empty interiors) in $\mathbb{R}^{n}$. A convex figure is said to be centered if it is symmetric about the origin, and a translate of a centered figure is said to be centrally symmetric. Let $\mathcal{C}_{s}^{n}$, and $\mathcal{K}_{s}^{n}$, denote the set of centrally symmetric figures in $\mathcal{C}^{n}$ and $\mathcal{K}^{n}$, respectively.

Let $G(n, i)$ denote the set of $i$-dimensional subspaces of $\mathbb{R}^{n}$. For $K \in \mathcal{K}^{n}$ and $\xi \in G(n, i)$, let $K \mid \xi$ denote the image of the orthogonal projection of $K$ onto $\xi$, and write $\operatorname{vol}_{i}(K \mid \xi)$ for the $i$-dimensional volume of $K \mid \xi$.

A theorem of Aleksandrov [1937] states that if $K, L \in \mathcal{K}_{s}^{n}$ and if for some $i$, such that $0<i<n$,

$$
\operatorname{vol}_{i}(K \mid \xi)=\operatorname{vol}_{i}(L \mid \xi), \quad \text { for all } \xi \in G(n, i),
$$

then

$L$ is a translate of $K$.

The following extension of Aleksandrov's theorem is a special case of Corollary (4.6) of this article:

Theorem. If $K \in \mathcal{K}_{s}^{n}, L \in \mathcal{K}^{n}$ and if for some $i$, such that $0<i<n$,

$$
\operatorname{vol}_{i}(K \mid \xi)=\operatorname{vol}_{i}(L \mid \xi), \quad \text { for all } \xi \in G(n, i),
$$

then,

$$
\operatorname{vol}_{n}(K) \geq \operatorname{vol}_{n}(L)
$$

with equality if and only if $L$ is a translate of $K$.

Received by the editors October 23, 1995.

1991 Mathematics Subject Classification. Primary 52A40.

Key words and phrases. Convex body, mixed volume, quermassintegral, zonoid, generalized zonoid, relative girth, relative brightness.

Research supported, in part, by NSF Grants DMS-9123571, and DMS-9507988.

(c)1997 American Mathematical Society 
Note that Aleksandrov's theorem is an immediate consequence: If $K, L$ are both in $\mathcal{K}_{s}^{n}$, and

$$
\operatorname{vol}_{i}(K \mid \xi)=\operatorname{vol}_{i}(L \mid \xi), \quad \text { for all } \xi \in G(n, i),
$$

then the Theorem gives both

$$
\operatorname{vol}_{n}(K) \geq \operatorname{vol}_{n}(L) \quad \text { and } \quad \operatorname{vol}_{n}(L) \geq \operatorname{vol}_{n}(K) .
$$

But the equality conditions of the inequality of the theorem now show that $K$ and $L$ must be translates of each other.

For the case $i=1$, the Theorem is well-known (it can safely be described as folklore). For the case $i=n-1$, the Theorem is due independently to Petty [1967] and Schneider [1967]. For $i=1$, the proof involves the use of Minkowski combinations and the Brunn-Minkowski inequality. For $i=n-1$, the proof involves the use of Blaschke combinations and the Kneser-Süss inequality. Unfortunately, for values of $i$ other than 1 and $n-1$ there is no analog of Minkowski and Blaschke combinations. Not only is no analog of these combinations known, but as Fedotov [1979] and Goodey \& Schneider [1980] have independently shown, none exists. The lack of a generalization of Minkowski and Blaschke combinations indicates the need for a new approach in establishing the Theorem. The methods presented in this article will work for all $i$.

\section{Notation And BACKGround Material}

For convex figures $K_{1}, \ldots, K_{r} \in \mathcal{C}^{n}$ and real numbers $\lambda_{1}, \ldots, \lambda_{r} \geq 0$, the Minkowski linear combination $\lambda_{1} K_{1}+\cdots+\lambda_{r} K_{r} \in \mathcal{C}^{n}$ is defined by

$$
\lambda_{1} K_{1}+\cdots+\lambda_{r} K_{r}=\left\{\lambda_{1} x_{1}+\cdots+\lambda_{r} x_{r} \in \mathbb{R}^{n}: x_{i} \in K_{i}\right\} .
$$

Of fundamental importance is the fact that the volume, $\operatorname{vol}_{n}\left(\lambda_{1} K_{1}+\cdots+\lambda_{r} K_{r}\right)$, of a linear combination of figures defined by (1.1), can be expressed as a symmetric homogeneous $n$-th degree polynomial in the $\lambda_{i}$ :

$$
\operatorname{vol}_{n}\left(\lambda_{1} K_{1}+\cdots+\lambda_{r} K_{r}\right)=\sum V_{i_{1} \cdots i_{n}} \lambda_{i_{1}} \cdots \lambda_{i_{n}},
$$

where the sum is taken over all $n$-tuples $\left(i_{1}, \ldots, i_{n}\right)$ of positive integers not exceeding $r$. The coefficient $V_{i_{1} \cdots i_{n}}$ (which is required to be symmetric in its subscripts) depends only on the figures $K_{i_{1}}, \ldots, K_{i_{n}}$, and is uniquely determined by (1.2); it is called the mixed volume of $K_{i_{1}}, \ldots, K_{i_{n}}$, and is written as $V\left(K_{i_{1}}, \ldots, K_{i_{n}}\right)$.

If $\xi \in G(n, r)$ and $K_{1}, \ldots, K_{r}$ are convex figures in $\xi$, then $v_{\xi}\left(K_{1}, \ldots, K_{r}\right)$ will be used to denote the $r$-dimensional mixed volume of the convex figures $K_{1}, \ldots, K_{r}$ in $\xi$. If $r=n-1$, then the subscript $\xi$, in $v_{\xi}\left(K_{1}, \ldots, K_{r}\right)$, will sometimes be suppressed.

If $K_{1}, \ldots, K_{n} \in \mathcal{C}^{n}$, and $\mathbf{C}=\left(K_{1}, \ldots, K_{n-1}\right)$, then $V\left(K_{1}, \ldots, K_{n}\right)$ will sometimes be abbreviated as $V\left(\mathbf{C}, K_{n}\right)$. The $i$-th Quermassintegral (or $i$-th mean projection measure) $W_{i}(K)$ of $K \in \mathcal{C}^{n}$ can be defined by

$$
W_{i}(K)=V(\underbrace{K, \ldots, K}_{n-i}, \underbrace{B, \ldots, B}_{i}) .
$$

Thus, $W_{0}(K)$ is the ordinary volume of $K$, while $n W_{1}(K)$ is the surface area of $K$.

Let $\delta$ denote the Hausdorff metric on $\mathcal{C}^{n}$; i.e., for $K, L \in \mathcal{C}^{n}$,

$$
\delta(K, L)=\min \{\varepsilon \geq 0: K \subset L+\varepsilon B \text { and } L \subset K+\varepsilon B\},
$$

where $B$ denotes the unit ball in $\mathbb{R}^{n}$. 
The following is a list of the needed basic properties of the mixed volume functional

$$
V: \underbrace{\mathcal{C}^{n} \times \cdots \times \mathcal{C}^{n}}_{n} \longrightarrow[0, \infty)
$$

(1.3) It is symmetric in its arguments.

(1.4) It is linear in each of its arguments with respect to Minkowski linear combinations; i.e., if $K_{1}, \ldots, K_{n-1} \in \mathcal{C}^{n}$, and $\mathbf{C}=\left(K_{1}, \ldots, K_{n-1}\right)$, then for $K, L \in \mathcal{C}^{n}$ and $\lambda, \mu \geq 0$,

$$
V(\mathbf{C}, \lambda K+\mu L)=\lambda V(\mathbf{C}, K)+\mu V(\mathbf{C}, L) .
$$

(1.5) Its diagonal form reduces to ordinary volume; i.e., for $K \in \mathcal{C}^{n}$,

$$
V(K, \ldots, K)=\operatorname{vol}_{n}(K) .
$$

(1.6) It is continuous (in fact, uniformly continuous) in each argument, with respect to the Hausdorff metric.

(1.7) It is invariant under independent translations of its arguments; i.e., if $K_{i} \in \mathcal{C}^{n}$, and $x_{i} \in \mathbb{R}^{n}$, then

$$
V\left(x_{1}+K_{1}, \ldots, x_{n}+K_{n}\right)=V\left(K_{1}, \ldots, K_{n}\right) .
$$

To state the next property needed, some additional notation is required. For $u \in$ $S^{n-1}$, let $\bar{u}$ denote the closed line segment joining the origin and $u$. If $x_{1}, \ldots, x_{r} \in$ $\mathbb{R}^{n}$, then write $\left[x_{1}, \ldots, x_{r}\right]$ for the linear space spanned by $x_{1}, \ldots, x_{r}$, and write $\left[x_{1}, \ldots, x_{r}\right]^{\perp}$ for the orthogonal complement of $\left[x_{1}, \ldots, x_{r}\right]$.

(1.8) If $K_{1}, \ldots, K_{r} \in \mathcal{C}^{n}$ and $u_{1}, \ldots, u_{n-r} \in S^{n-1}$, and $\xi=\left[u_{1}, \ldots, u_{n-r}\right]^{\perp}$ then

$$
V\left(K_{1}, \ldots, K_{r}, \bar{u}_{1}, \ldots, \bar{u}_{n-r}\right)=c_{r} v_{\xi}\left(K_{1}\left|\xi, \ldots, K_{r}\right| \xi\right) \operatorname{vol}_{n-r}\left(\bar{u}_{1}+\cdots+\bar{u}_{n-r}\right),
$$

where $c_{r}$ depends only on $r$ (and $n$ ). This well known (see, e.g. Burago-Zalgaller [1988]) and useful fact is an easy consequence of (5.3.23) in Schneider [1993].

One of the fundamental inequalities for mixed volumes is the Minkowski (mixed volume) inequality: If $K, L \in \mathcal{K}^{n}$, then

$$
V(\underbrace{K, \ldots, K}_{n-1}, L)^{n} \geq \operatorname{vol}_{n}(K)^{n-1} \operatorname{vol}_{n}(L),
$$

with equality if and only if $K$ and $L$ are homothetic.

The Aleksandrov-Fenchel inequality states that if $K_{1}, \ldots, K_{n} \in \mathcal{C}^{n}$, then

$$
V\left(K_{1}, \ldots, K_{n}\right)^{r} \geq \prod_{j=1}^{r} V(\underbrace{K_{j}, \ldots, K_{j}}_{r}, K_{r+1}, \ldots, K_{n}) .
$$

Unfortunately, the equality conditions in the Aleksandrov-Fenchel inequality are only known when restrictions are imposed on the bodies involved (see e.g. Schneider [1985], [1990]). In this article, the only equality conditions used are for the case where $K_{1}=\cdots=K_{r-1}$, and $K_{r+1}=\cdots=K_{n}=B$. Specifically, if $K, L \in \mathcal{K}^{n}$ and $0 \leq i<n-1$, then

$$
V(\underbrace{K, \ldots, K}_{n-i-1}, \underbrace{B, \ldots, B}_{i}, L)^{n-i} \geq W_{i}(K)^{n-i-1} W_{i}(L),
$$

with equality if and only if $K$ and $L$ are homothetic. See Leichtweiß [1980]. Of course, the case $i=0$ of this inequality is just the Minkowski inequality. 
A zonotope is a Minkowski combination of line segments; i.e., $Z \in \mathcal{C}^{n}$ is a zonotope if there exist $u_{1}, \ldots, u_{r} \in S^{n-1}$ and $\lambda_{1}, \ldots, \lambda_{r} \geq 0$, such that

$$
Z=\lambda_{1} \bar{u}_{1}+\cdots+\lambda_{r} \bar{u}_{r} \text {. }
$$

A body in $\mathcal{C}^{n}$ that is the limit (with respect to the Hausdorff metric) of zonotopes is called a zonoid, and $\mathcal{Z}^{n}$ will be used to denote the class of zonoids. It is easily verified that $\mathcal{Z}^{n} \subset \mathcal{C}_{s}^{n}$. A body $K \in \mathcal{C}^{n}$ is said to be a generalized zonoid if there exists a $Z \in \mathcal{Z}^{n}$, such that $K+Z \in \mathcal{Z}^{n}$. While the class of generalized zonoids, $\mathcal{Z}_{g}^{n}$, is a proper subset of $\mathcal{C}_{s}^{n}$, it turns out that $\mathcal{Z}_{g}^{n}$ is dense in $\mathcal{C}_{s}^{n}$. For reference regarding zonoids and generalized zonoids, the reader is referred to the excellent surveys of Schneider \& Weil [1983] and Goodey \& Weil [1993], and the book of Schneider [1993].

\section{AN IDENTITY FOR MiXed VOLUMES}

The following easy result will be one of the main ingredients in the proof of the Theorem stated in the introduction. It can be deduced from mixed volume formulas in Weil [1974], [1976], [1979], and Schneider \& Weil [1983]. The techniques used in the proof are from the previously cited work of Weil.

Lemma (2.1). If $1 \leq r<n$ and $K_{1}, K_{1}^{\prime}, \ldots, K_{r}, K_{r}^{\prime} \in \mathcal{C}^{n}$, while $\mathbf{C}=\left(K_{1}, \ldots, K_{r}\right)$ and $\mathbf{C}^{\prime}=\left(K_{1}^{\prime}, \ldots, K_{r}^{\prime}\right)$, then

$$
V\left(\mathbf{C}, \bar{u}_{r+1}, \ldots, \bar{u}_{n}\right)=V\left(\mathbf{C}^{\prime}, \bar{u}_{r+1}, \ldots, \bar{u}_{n}\right), \quad \text { for all } u_{i} \in S^{n-1},
$$

if and only if

$$
V\left(\mathbf{C}, Q_{r+1}, \ldots, Q_{n}\right)=V\left(\mathbf{C}^{\prime}, Q_{r+1}, \ldots, Q_{n}\right), \quad \text { for all } Q_{i} \in \mathcal{K}_{s}^{n} .
$$

Proof. From (1.3) it follows that to show that (2.1.1) implies (2.1.2), all that need be demonstrated is that

$$
V\left(\mathbf{C}, \bar{u}_{r+1}, \ldots, \bar{u}_{n-1}, \bar{u}\right)=V\left(\mathbf{C}^{\prime}, \bar{u}_{r+1}, \ldots, \bar{u}_{n-1}, \bar{u}\right),
$$

for all $u \in S^{n-1}$, implies that

$$
V\left(\mathbf{C}, \bar{u}_{r+1}, \ldots, \bar{u}_{n-1}, Q\right)=V\left(\mathbf{C}^{\prime}, \bar{u}_{r+1}, \ldots, \bar{u}_{n-1}, Q\right),
$$

for all $Q \in \mathcal{K}_{s}^{n}$. To see this, note that from the definition of a zonotope (1.12), and the Minkowski linearity of mixed volumes (1.4), it follows that (2.2.1) implies

$$
V\left(\mathbf{C}, \bar{u}_{r+1}, \ldots, \bar{u}_{n-1}, Z\right)=V\left(\mathbf{C}^{\prime}, \bar{u}_{r+1}, \ldots, \bar{u}_{n-1}, Z\right),
$$

for all zonotopes $Z$. The continuity of mixed volumes (1.6) and the definition of a zonoid shows that (2.3) must hold for all $Z \in \mathcal{Z}^{n}$.

Suppose $Q \in \mathcal{Z}_{g}^{n}$. Thus there exists a $Z \in \mathcal{Z}^{n}$ such that $Q+Z \in \mathcal{Z}^{n}$. Let $\mathbf{D}=\left(K_{1}, \ldots, K_{r}, \bar{u}_{r+1}, \ldots, \bar{u}_{n-1}\right)$ and $\mathbf{D}^{\prime}=\left(K_{1}^{\prime}, \ldots, K_{r}^{\prime}, \bar{u}_{r+1}, \ldots, \bar{u}_{n-1}\right)$. Since $Q+Z \in \mathcal{Z}^{n}$, it follows from (2.3), and the Minkowski linearity of mixed volumes (1.4), that

$$
V(\mathbf{D}, Q)+V(\mathbf{D}, Z)=V(\mathbf{D}, Q+Z)=V\left(\mathbf{D}^{\prime}, Q+Z\right)=V\left(\mathbf{D}^{\prime}, Q\right)+V\left(\mathbf{D}^{\prime}, Z\right) .
$$

Since by $(2.3), V(\mathbf{D}, Z)=V\left(\mathbf{D}^{\prime}, Z\right)$, it follows that $V(\mathbf{D}, Q)=V\left(\mathbf{D}^{\prime}, Q\right)$, showing that (2.2.2) holds whenever $Q$ is a generalized zonoid. Since every member of $\mathcal{K}_{s}^{n}$ is the limit of generalized zonoids, it follows from the continuity of mixed volumes (1.6), that (2.2.2) must hold for all $Q \in \mathcal{K}_{s}^{n}$.

That (2.1.2) implies (2.1.1) is trivial: For each $i$, choose a sequence $Q_{i, j} \in \mathcal{K}_{s}^{n}$, such that $\lim _{j \rightarrow \infty} Q_{i, j}=\bar{u}_{i}$, and use the continuity of mixed volumes (1.6). 


\section{Bodies OF CONSTANT RELATIVE GIRTH}

Suppose $M \in \mathcal{K}_{s}^{n}$, and $0<r<n-1$. Following Chakerian [1967], a body $K \in \mathcal{K}^{n}$ is said to have constant relative $r$-girth, equal to $c$, with respect to $M$, if the $(n-1)$-dimensional mixed volume

$$
v(\underbrace{K\left|u^{\perp}, \ldots, K\right| u^{\perp}}_{r}, \underbrace{M\left|u^{\perp}, \ldots, M\right| u^{\perp}}_{n-r-1})=c \operatorname{vol}_{n-1}\left(M \mid u^{\perp}\right),
$$

for all $u \in S^{n-1}$. In general, the bodies $K_{1}, \ldots, K_{r} \in \mathcal{K}^{n}$ are said to have constant relative mixed $r$-girth, equal to $c$, with respect to $M$, if the $(n-1)$-dimensional mixed volume

$$
v(K_{1}\left|u^{\perp}, \ldots, K_{r}\right| u^{\perp}, \underbrace{M\left|u^{\perp}, \ldots, M\right| u^{\perp}}_{n-r-1})=c \operatorname{vol}_{n-1}\left(M \mid u^{\perp}\right),
$$

for all $u \in S^{n-1}$.

The following characterization will be needed:

Lemma (3.2). The bodies $K_{1}, \ldots, K_{r} \in \mathcal{K}^{n}$ have constant relative mixed $r$-girth, equal to $c$, with respect to $M \in \mathcal{K}_{s}^{n}$, if and only if

$$
V(K_{1}, \ldots, K_{r}, \underbrace{M, \ldots, M}_{n-r-1}, Q)=c V(\underbrace{M, \ldots, M}_{n-1}, Q),
$$

for all $Q \in \mathcal{K}_{s}^{n}$.

Proof. From definition (3.1), and property (1.8), it follows that $K_{1}, \ldots, K_{r}$ have constant relative $r$-girth, equal to $c$, with respect to $M$, if and only if,

$$
V(K_{1}, \ldots, K_{r}, \underbrace{M, \ldots, M}_{n-r-1}, \bar{u})=c V(\underbrace{M, \ldots, M}_{n-1}, \bar{u}),
$$

for all $u \in S^{n-1}$. But Lemma (2.1) shows that this is equivalent to condition $(3.2 .1)$.

Obviously, from Lemma (3.2), a body $K \in \mathcal{K}^{n}$ has constant relative $r$-girth, equal to $c$, with respect to $M \in \mathcal{K}_{s}^{n}$, if and only if

$$
V(\underbrace{K, \ldots, K}_{r}, \underbrace{M, \ldots, M}_{n-r-1}, Q)=c V(\underbrace{M, \ldots, M}_{n-1}, Q),
$$

for all $Q \in \mathcal{K}_{s}^{n}$.

By choosing $Q=M$ in Lemma (3.2), using (1.5), Lemma (3.2) becomes

Lemma (3.3). The bodies $K_{1}, \ldots, K_{r} \in \mathcal{K}^{n}$ have constant relative mixed $r$-girth, with respect to $M \in \mathcal{K}_{s}^{n}$, if and only if

$$
\begin{aligned}
\operatorname{vol}_{n}(M) & V(K_{1}, \ldots, K_{r}, \underbrace{M, \ldots, M}_{n-r-1}, Q) \\
& =V(K_{1}, \ldots, K_{r}, \underbrace{M, \ldots, M}_{n-r}) V(\underbrace{M, \ldots, M}_{n-1}, Q),
\end{aligned}
$$

for all $Q \in \mathcal{K}_{s}^{n}$. 
Hence, the body $K \in \mathcal{K}^{n}$ has constant relative $r$-girth, with respect to the body $M \in \mathcal{K}_{s}^{n}$, if and only if

$\operatorname{vol}_{n}(M) V(\underbrace{K, \ldots, K}_{r}, \underbrace{M, \ldots, M}_{n-r-1}, Q)=V(\underbrace{K, \ldots, K}_{r}, \underbrace{M, \ldots, M}_{n-r}) V(\underbrace{M, \ldots, M}_{n-1}, Q)$,

for all $Q \in \mathcal{K}_{s}^{n}$.

Theorem (3.4). If $K_{1}, \ldots, K_{r} \in \mathcal{K}^{n}$ have constant unit relative mixed $r$-girth, with respect to $M \in \mathcal{K}_{s}^{n}$, then

$$
\operatorname{vol}_{n}(M)^{r} \geq \operatorname{vol}_{n}\left(K_{1}\right) \cdots \operatorname{vol}_{n}\left(K_{r}\right),
$$

and

$$
W_{1}(M)^{r} \geq W_{1}\left(K_{1}\right) \cdots W_{1}\left(K_{r}\right),
$$

with equality in either inequality implying that the $K_{i}$ are homothetic to $M$.

Proof. In Lemma (3.2) take $Q=M$, use (1.5), and get

$$
\operatorname{vol}_{n}(M)=V(K_{1}, \ldots, K_{r}, \underbrace{M, \ldots, M}_{n-r}) .
$$

The Aleksandrov-Fenchel inequality (1.10), together with (1.5), applied to the mixed volume above yields

$$
\operatorname{vol}_{n}(M)^{n-1} \geq \operatorname{vol}_{n}(M)^{n-r-1} \prod_{i=1}^{r} V(\underbrace{K_{i}, \ldots, K_{i}}_{n-1}, M) .
$$

The Minkowski inequality (1.9), applied to the mixed volumes, will now yield inequality (3.4.1), with its equality conditions.

In Lemma (3.2) take $Q=B$, and from the definition of $W_{1}(M)$ get

$$
W_{1}(M)=V(K_{1}, \ldots, K_{r}, \underbrace{M, \ldots, M}_{n-r-1}, B) .
$$

When the Aleksandrov-Fenchel inequality (1.10) is applied to the mixed volume on the right, the result is:

$$
W_{1}(M)^{n-2} \geq W_{1}(M)^{n-r-2} \prod_{i=1}^{r} V(\underbrace{K_{i}, \ldots, K_{i}}_{n-2}, M, B) .
$$

Inequality (1.11), applied to the mixed volumes, will now yield inequality (3.4.2), with its equality conditions.

If in Theorem (3.4) $K_{1}=\cdots=K_{r}$, then the equality conditions are stronger:

Corollary (3.5). If $K \in \mathcal{K}^{n}$ has constant unit relative $r$-girth, with respect to $M \in \mathcal{K}_{s}^{n}$, then

$$
\operatorname{vol}_{n}(M) \geq \operatorname{vol}_{n}(K) \quad \text { and } \quad W_{1}(M) \geq W_{1}(K),
$$

with equality in either inequality if and only if $K$ is a translate of $M$. 


\section{Bodies of CONSTANT Relative BRightness}

Suppose $M \in \mathcal{K}_{s}^{n}$, and $0<r<n-1$. A body $K \in \mathcal{K}^{n}$ is said to have constant relative $r$-brightness, equal to $c$, with respect to $M$, if

$$
\operatorname{vol}_{r}(K \mid \xi)=c \operatorname{vol}_{r}(M \mid \xi) \quad \text { for all } \xi \in G(n, r) .
$$

In general, the bodies $K_{1}, \ldots, K_{r} \in \mathcal{K}^{n}$ are said to have constant relative mixed $r$-brightness, equal to $c$, with respect to $M$, if

$$
v_{\xi}\left(K_{1}\left|\xi, \ldots, K_{r}\right| \xi\right)=c \operatorname{vol}_{r}(M \mid \xi) \quad \text { for all } \xi \in G(n, r) .
$$

The following characterization will be used:

Lemma (4.2). The bodies $K_{1}, \ldots, K_{r} \in \mathcal{K}^{n}$ have constant relative mixed $r$ brightness, equal to $c$, with respect to $M \in \mathcal{K}_{s}^{n}$, if and only if

$$
V\left(K_{1}, \ldots, K_{r}, Q_{1}, \ldots, Q_{n-r}\right)=c V(\underbrace{M, \ldots, M}_{r}, Q_{1}, \ldots, Q_{n-r}),
$$

for all $Q_{i} \in \mathcal{K}_{s}^{n}$.

Proof. First, suppose $K_{1}, \ldots, K_{r}$ have constant relative mixed $r$-brightness, equal to $c$, with respect to $M$. If $u_{1}, \ldots, u_{n-r} \in S^{n-1}$, then by (1.8),

$$
V\left(K_{1}, \ldots, K_{r}, \bar{u}_{1}, \ldots, \bar{u}_{n-r}\right)=c_{r} v_{\xi}\left(K_{1}\left|\xi, \ldots, K_{r}\right| \xi\right) \operatorname{vol}_{n-r}\left(\bar{u}_{1}+\cdots+\bar{u}_{n-r}\right),
$$

where $\xi=\left[u_{1}, \ldots, u_{n-r}\right]^{\perp}$. Also by (1.8),

$$
V(\underbrace{M, \ldots, M}_{r}, \bar{u}_{1}, \ldots, \bar{u}_{n-r})=c_{r} \operatorname{vol}_{r}(M \mid \xi) \operatorname{vol}_{n-r}\left(\bar{u}_{1}+\cdots+\bar{u}_{n-r}\right) .
$$

Since by (4.1),

$$
v_{\xi}\left(K_{1}\left|\xi, \ldots, K_{r}\right| \xi\right)=c \operatorname{vol}_{r}(M \mid \xi)
$$

it follows that

$$
V\left(K_{1}, \ldots, K_{r}, \bar{u}_{1}, \ldots, \bar{u}_{n-r}\right)=c V(\underbrace{M, \ldots, M}_{r}, \bar{u}_{1}, \ldots, \bar{u}_{n-r}),
$$

from which Lemma (2.1) yields (4.2).

Now, suppose (4.2) holds, and suppose $\xi \in G(n, r)$. Choose $u_{1}, \ldots, u_{n-r} \in S^{n-1}$ such that $\left[u_{1}, \ldots, u_{n-r}\right]=\xi^{\perp}$. For each $i$, choose a sequence $Q_{i, j} \in \mathcal{K}_{s}^{n}$, such that $\lim _{j \rightarrow \infty} Q_{i, j}=\bar{u}_{i}$. From (4.2), and the continuity of mixed volumes (1.6), it follows that

$$
V\left(K_{1}, \ldots, K_{r}, \bar{u}_{1}, \ldots, \bar{u}_{n-r}\right)=c V(\underbrace{M, \ldots, M}_{r}, \bar{u}_{1}, \ldots, \bar{u}_{n-r}) .
$$

But by (1.8) this implies that

$$
v_{\xi}\left(K_{1}\left|\xi, \ldots, K_{r}\right| \xi\right)=c \operatorname{vol}_{r}(M \mid \xi),
$$

showing that $K_{1}, \ldots, K_{r}$ have constant relative mixed $r$-brightness, equal to $c$, with respect to $M$.

Thus, a body $K \in \mathcal{K}^{n}$ has constant relative $r$-brightness, equal to $c$, with respect to $M \in \mathcal{K}_{s}^{n}$, if and only if

$$
V(\underbrace{K, \ldots, K}_{r}, Q_{1}, \ldots, Q_{n-r})=c V(\underbrace{M, \ldots, M}_{r}, Q_{1}, \ldots, Q_{n-r}),
$$

for all $Q_{i} \in \mathcal{K}_{s}^{n}$. 
By choosing $Q_{1}=\cdots=Q_{n-r}=M$ in Lemma (4.2), and using (1.5), Lemma (4.2) can be rewritten as:

Lemma (4.3). The bodies $K_{1}, \ldots, K_{r} \in \mathcal{K}^{n}$ have constant relative mixed $r$ brightness, with respect to $M \in \mathcal{K}_{s}^{n}$, if and only if

$$
\begin{aligned}
& \operatorname{vol}_{n}(M) V\left(K_{1}, \ldots, K_{r}, Q_{1}, \ldots, Q_{n-r}\right) \\
& \quad=V(K_{1}, \ldots, K_{r}, \underbrace{M, \ldots, M}_{n-r}) V(\underbrace{M, \ldots, M}_{r}, Q_{1}, \ldots, Q_{n-r}),
\end{aligned}
$$

for all $Q_{i} \in \mathcal{K}_{s}^{n}$.

Thus, a body $K \in \mathcal{K}^{n}$ has constant relative $r$-brightness, with respect to $M \in$ $\mathcal{K}_{s}^{n}$, if and only if

$$
\begin{aligned}
\operatorname{vol}_{n}(M) V(\underbrace{K, \ldots, K}_{r}, Q_{1}, \ldots, Q_{n-r}) \\
=V(\underbrace{K, \ldots, K}_{r}, \underbrace{M, \ldots, M}_{n-r}) V(\underbrace{M, \ldots, M}_{r}, Q_{1}, \ldots, Q_{n-r}),
\end{aligned}
$$

for all $Q_{i} \in \mathcal{K}_{s}^{n}$.

By choosing $Q_{1}=\cdots=Q_{n-r-1}=M$ in Lemma (4.2), it follows from Lemma (3.2) that:

Proposition (4.4). If $K_{1}, \ldots, K_{r} \in \mathcal{K}^{n}$ have constant relative mixed $r$-brightness, with respect to $M \in \mathcal{K}_{s}^{n}$ then $K_{1}, \ldots, K_{r} \in \mathcal{K}^{n}$ have constant relative mixed r-girth, with respect to $M$.

Thus, if $K \in \mathcal{K}^{n}$ has constant relative $r$-brightness, with respect to $M \in \mathcal{K}_{s}^{n}$ then $K \in \mathcal{K}^{n}$ has constant relative $r$-girth, with respect to $M$. For the case where $M=B$, this was established by Firey [1970].

Theorem (4.5). If $M \in \mathcal{K}_{s}^{n}$, and $K_{1}, \ldots, K_{r} \in \mathcal{K}^{n}$, and if

$$
v_{\xi}\left(K_{1}\left|\xi, \ldots, K_{r}\right| \xi\right)=\operatorname{vol}_{r}(M \mid \xi), \quad \text { for all } \xi \in G(n, r),
$$

then

$$
W_{n-r}(M)=V(K_{1}, \ldots, K_{r}, \underbrace{B, \ldots, B}_{n-r}),
$$

and for all $j$ such that $0 \leq j<n-r$,

$$
W_{j}(M)^{r} \geq W_{j}\left(K_{1}\right) \cdots W_{j}\left(K_{r}\right),
$$

with equality, for any $j$, implying that the $K_{i}$ are all homothetic to $M$.

Proof. Taking $Q_{1}=\cdots=Q_{n-r}=B$ in Lemma (4.2), gives

$$
W_{n-r}(M)=V(K_{1}, \ldots, K_{r}, \underbrace{B, \ldots, B}_{n-r}) \text {. }
$$

Suppose $0 \leq j<n-r$. Take $Q_{1}=\cdots=Q_{j}=B$ and $Q_{j+1}=\cdots=Q_{n-r}=M$ in Lemma (4.2), and get

$$
W_{j}(M)=V(K_{1}, \ldots, K_{r}, \underbrace{M, \ldots, M}_{n-r-j}, \underbrace{B, \ldots, B}_{j}) .
$$


The Aleksandrov-Fenchel inequality (1.10) is applied to the mixed volume on the right to give:

$$
W_{j}(M)^{n-j-1} \geq W_{j}(M)^{n-j-r-1} \prod_{i=1}^{r} V(\underbrace{K_{i}, \ldots, K_{i}}_{n-j-1}, M, \underbrace{B, \ldots, B}_{j}) .
$$

Inequality (1.11), the Aleksandrov-Fenchel inequality with equality conditions, applied to the mixed volumes in the product, gives

$$
W_{j}(M)^{r} \geq W_{j}\left(K_{1}\right) \cdots W_{j}\left(K_{r}\right),
$$

with equality implying that each $K_{i}$ is homothetic to $M$.

For the special case of Theorem (4.5) where $K_{1}=\cdots=K_{r}$, the equality conditions are stronger:

Corollary (4.6). If $M \in \mathcal{K}_{s}^{n}$, and $K \in \mathcal{K}^{n}$, and if

$$
\operatorname{vol}_{r}(K \mid \xi)=\operatorname{vol}_{r}(M \mid \xi), \quad \text { for all } \xi \in G(n, r),
$$

then $W_{n-r}(M)=W_{n-r}(K)$, and for all $j$ such that $0 \leq j<n-r$,

$$
W_{j}(M) \geq W_{j}(K),
$$

with equality, for any $j$, if and only if $K$ is a translate of $M$.

Let $\mathcal{V}_{r}(M)$ denote the set of all bodies in $\mathcal{K}^{n}$ with constant relative $r$-brightness, with respect to $M$.

Corollary (4.7). The only centrally symmetric bodies in $\mathcal{V}_{r}(M)$ are homothetic to $M$.

\section{REFERENCES}

[1937] A. D. Aleksandrov, On the theory of mixed volumes. II. New inequalities between mixed volumes and their application, Mat. Sbornik N.S. 2 (1937), 1205-1238, Russian.

[1988] Yu. D. Burago and V. A. Zalgaller, Geometric Inequalities, Springer Verlag, Heidelberg, 1988. MR 89b:52020

[1967] G. D. Chakerian, Sets of constant relative width and constant relative brightness, Trans. Amer. Math. Soc. 129 (1967), 26-37. MR 35:3545

[1979] V. P. Fedotov, A counterexample to a hypothesis of Firey, Math. Zametki 26 (1979), 269-275, Russian. MR 80i:52009

[1970] Wm. J. Firey, Convex bodies of constant outer p-measure, Mathematika 17 (1970), 21-27. MR 42:2367

[1980] P. R. Goodey and R. Schneider, On intermediate area functions of convex bodies, Math. Z. 173 (1980), 185-194. MR 81k:52010

[1993] P. R. Goodey and W. Weil, Zonoids and generalizations, Handbook of Convex Geometry (P.M. Gruber and J.M. Wills, Eds.), North-Holland, Amsterdam, 1993. MR 95g:52015

[1980] K. Leichtweiß, Konvexe Mengen, Springer, Berlin, 1980. MR 81j:52001

[1967] C. M. Petty, Projection bodies, Proc. Coll. Convexity, Copenhagen, 1965, Københavns Univ. Mat. Inst., 1967, pp. 234-241. MR 35:7203

[1967] R. Schneider, Zur einem Problem von Shephard über die Projektionen konvexer Körper, Math. Z. 101 (1967), 71-82. MR 36:2059

[1985] R. Schneider, On the Aleksandrov-Fenchel inequality, Ann. N.Y. Acad. Sci. 440 (1985), 132-141. MR 87c:52019

[1990] R. Schneider, On the Aleksandrov-Fenchel inequality for convex bodies, Results in Math. 17 (1990), 287-295. MR 91b:52009

[1993] R. Schneider, Convex Bodies: The Brunn-Minkowski Theory, Cambridge U. Press, Cambridge, 1993. MR 94d:52007 
[1983] R. Schneider and W. Weil, Zonoids and related topics, Convexity and its Applications (P.M. Gruber and J.M. Wills, Eds.), Birkhäuser, Basel, 1983, pp. 296-317. MR 85c:52010

[1974] W. Weil, Über den Vektorraum der Differenzen von Stützfunktionen konvexer Körper, Math. Nachr. 59 (1974), 353-369. MR 49:6033

[1976] W. Weil, Kontinuierliche Linearkombination von Strecken, Math. Z. 148 (1976), 71-84. MR 53:3887

[1979] W. Weil, Centrally symmetric convex bodies and distributions II, Israel J. Math. 32 (1979), 143-182. MR 80g:52003

Department of Mathematics, University of California, Davis, California 95616

Department of Applied Mathematics and Physics, Polytechnic University, Brooklyn, NEW YORK 11201

E-mail address: lutwak@magnus.poly.edu 